\title{
Interval Type-2 Fuzzy Multi Criteria Decision Making Based on Intuitive Multiple Centroid
}

\author{
Ku Muhammad Naim Ku Khalif ${ }^{1}$, Alexander Gegov ${ }^{2}$, Ahmad Syafadhli Abu \\ Bakar $^{3,4}$, Noor Zuraidin Mohd Safar ${ }^{5}$ \\ ${ }^{1}$ Centre for Mathematical Sciences, Universiti Malaysia Pahang, Malaysia \\ ${ }^{2}$ School of Computing, University of Portsmouth, United Kingdom \\ ${ }^{3}$ Centre for Foundation Studies in Science, Universiti Malaya, Malaysia \\ ${ }^{4}$ Centre of Research for Computational Sciences and Informatics in Biology, Bioindustry, \\ Environment, Agriculture and Healthcare (CRYSTAL), Universiti Malaya, Malaysia \\ ${ }^{5}$ Faculty of Computer Science and Information Technology, Universiti Tun Hussien Onn \\ Malaysia, Malaysia \\ kunaim@ump.edu.my, alexander.gegov@port.ac.uk, ahmadsyafadhli@um.edu.my, \\ zuraidin@uthm.edu.my
}

\begin{abstract}
This paper aims to introduce fuzzy multi criteria decision making model using consistent fuzzy preference relations and fuzzy technique for order performance by similarity to ideal solution sets that is incorporated with intuitive multiple centroid defuzzification in the context of interval type- 2 fuzzy. The implementation of interval type-2 fuzzy sets is taken into consideration, where it has more authority to provide more degree of freedom in representing the uncertainty of human based decision making problems. It also highlights the combination of interval type-2 fuzzy sets with multi criteria decision making techniques allow the use of fuzzy linguistic by considering the need of human intuition in decision making problems under uncertain environment. Numerical example is included to illustrate the proposed model. The proposed model is importantly needed to validate using sensitivity analysis in order to analyse the quality and robustness of the model in giving the most promising alternative with respect to resources. The results show that it is highly practical to use the proposed model in decision making evaluation.
\end{abstract}

Keywords: Fuzzy multi criteria decision making, Consistent fuzzy preference relations, Fuzzy TOPSIS, Interval type-2 fuzzy sets, Intuitive multiple centroid, uncertainty

\section{Introduction}

Uncertainty and fuzziness are well-known phenomena in many applications areas in science and engineering, where are often not crisp but there exist various degree of membership grade that practical automatically occurs in decision making problems. Type-2 fuzzy sets are appropriately tools for uncertainty or approximate reasoning modelling. It has more authority to provide more degree of freedom in representing the uncertainty of human based decision making problems. Klir and Yuan claim that 
the type-1 fuzzy sets inly describe imprecision not uncertainty [1]. On particular motivation for the further interest in type-2 fuzzy sets that its' provide a better scope for modelling uncertainty than type-1 fuzzy sets [2]. According to Karnik and Mendel, they claim that type-2 fuzzy sets can be characterised as fuzzy membership function where the membership value for type-2 fuzzy sets is in interval form $[0,1]$, unlike type-1 fuzzy sets where the membership value is a crisp value in $[0,1][3]$.

Defuzzification plays important role in the performance of fuzzy systems' modelling techniques. Defuzzification process is guided by the output fuzzy subset that one value would be selected as a single crisp value as the system output. While much of the literature discuss there are variety of defuzzification methods have largely developed. Though, each of them have difference performance in difference applications and there is a general method can satisfy the performance in all conditions which is centroid method [4]. Centroid defuzzification methods of fuzzy numbers have been explored for the last decade that commonly used and have been applied in various discipline areas. The computational complexity of type-2 fuzzy sets is very difficult to handle into practical applications because of characterised by their footprint of uncertainty [5].

In literature, most of the hybrid MCDM model combined two techniques in order to tackle the evaluation of criteria and the evaluation of alternatives respectively. The evaluation process of criteria and alternatives play important role in MCDM techniques requirements. To identify the best decision to be made among the various alternatives with several criteria, the methodology has to study the preferences among the criteria to make sure the weights of criteria are reliable enough to be implemented in the selection of alternatives. In this paper, the hybrid of consistent fuzzy preference relations and fuzzy technique for order of preference by similarity to ideal solution (TOPSIS) using new centroid defuzzification for interval type-2 fuzzy sets is proposed in dealing with uncertainty events. The major weaknesses of classical TOPSIS are in not providing for weight elicitation, and consistency checking for judgments' evaluation. Hence, in this paper, the authors consider the fuzzy TOPSIS's employment that has been significantly restrained by the human capacity for information processing. Sensitivity analysis [6] is applied to validate the proposed model. It can effectively contributes to making accurate decisions by assuming that a set of weights for criteria or alternatives then obtained a new round of weights for them, so that the efficiency of alternatives has become equal or their order has changed.

The rest of this paper is organised as follows: Section 2 discusses the theoretical preliminaries of fuzzy set theory and generalised trapezoidal fuzzy numbers. This is then preceded to the proposed work of integrated fuzzy MCDM model that consist of consistent fuzzy preference relations and fuzzy TOPSIS using intuitive multiple centroid defuzzification in Section 3. Section 4 discusses the case study and results that illustrated the proposed model and validation processes using sensitivity analysis. Finally, Section 5 gives the conclusion. 


\section{Theoretical Preliminaries}

In this section, the authors briefly review some definitions of interval type-2 fuzzy set that are illustrated as follows.

A. Interval Type-2 Fuzzy Set

Definition 1[7]: A type-2 fuzzy set $\tilde{A}$ in the universe of discourse $X$ represented by the type-2 membership function, $\mu$. If all $\mu_{\tilde{\tilde{A}}}(x, u)=1$, then $\tilde{A}$ is called an interval type-2 fuzzy sets. An interval type-2 fuzzy set can be considered as a special case of type-2 fuzzy sets, denoted as follows.

$$
\tilde{\tilde{A}}=\int_{x \in X} \int_{u \in J_{x}} 1 /(x, u), \text { where } J_{x} \subseteq[0,1]
$$

Definition 2 [7]: The upper and lower membership functions of an interval type-2 fuzzy set are type-1 fuzzy sets membership functions, respectively. A trapezoidal interval type-2 fuzzy set can be represented by, $\tilde{\tilde{A}}_{i}=\left(\tilde{A}_{i}^{U}, \tilde{A}_{i}^{L}\right)=\left(\left(a_{i 1}^{U}, a_{i 2}^{U}, a_{i 3}^{U}, a_{i 4}^{U} ; H_{1}\left(\tilde{A}_{i}^{U}\right), \tilde{H}_{2}\left(\tilde{A}_{i}^{L}\right)\right),\left(a_{i 1}^{L}, a_{i 2}^{L}, a_{i 3}^{L}, a_{i 4}^{L} ; H_{1}\left(\tilde{A}_{i}^{L}\right), H_{2}\left(\tilde{A}_{i}^{L}\right)\right)\right)$ where can be depicted in Fig. 1 [8]. The $\tilde{A}_{i}^{U}$ and $\tilde{A}_{i}^{L}$ are type-1 fuzzy sets, $a_{i 1}^{U}, a_{i 2}^{U}, a_{i 3}^{U}, a_{i 4}^{U}, a_{i 1}^{L}, a_{i 2}^{L}, a_{i 3}^{L}$ and $a_{i 4}^{L}$ are the reference points of the interval type-2 fuzzy sets $\tilde{\tilde{A}}, H_{j}\left(\tilde{A}_{i}^{U}\right)$ denote the membership value of the element $a_{i(j+1)}^{U}$ in the upper trapezoidal membership function $\tilde{A}_{i}^{U}, 1 \leq j \leq 2, H_{j}\left(\tilde{A}_{i}^{L}\right)$ denotes the membership value of the element $a_{i(j+1)}^{L}$ in the lower trapezoidal membership function $\tilde{A}_{i}^{L}, 1 \leq j \leq 2$, and for $H_{1}\left(\tilde{A}_{i}^{U}\right) \in[0,1], H_{2}\left(\tilde{A}_{i}^{U}\right) \in[0,1], \quad H_{1}\left(\tilde{A}_{i}^{L}\right) \in[0,1]$, $H_{2}\left(\tilde{A}_{i}^{L}\right) \in[0,1]$ and $1 \leq i \leq n, H_{2}\left(\tilde{A}_{i}^{U}\right) \in[0,1]$.

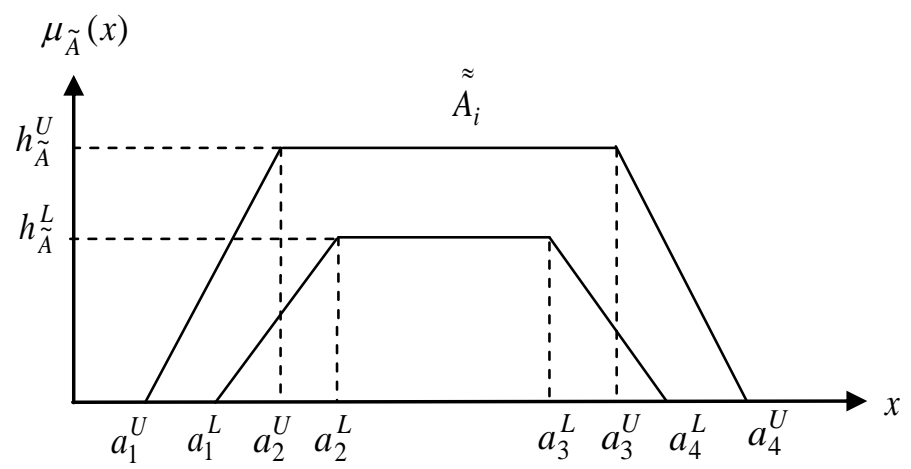

Fig. 1. The representation of interval type-2 fuzzy set. 


\section{Proposed Model}

This section focuses on the development of fuzzy MCDM model that is incorporated with intuitive multiple centroid for interval type-2 fuzzy sets.

\section{Step 1: Determine the weights of evaluation criteria.}

The weighting of evaluation criteria are employed.

Step 2: Construct a pairwise comparison matrices.

The pairwise comparison matrices are constructed among all criteria in the dimension of the hierarchy systems based on the decision makers' preferences using following matrix:

$$
A=\left[\begin{array}{cccc}
1 & \tilde{a}_{12} & \cdots & \tilde{a}_{1 n} \\
\tilde{a}_{21} & 1 & \cdots & \tilde{a}_{2 n} \\
\vdots & \vdots & \ddots & \vdots \\
\tilde{a}_{n 1} & \tilde{a}_{n 2} & \cdots & 1
\end{array}\right]=\left[\begin{array}{cccc}
1 & \tilde{a}_{12} & \cdots & \tilde{a}_{1 n} \\
1 / \tilde{a}_{12} & 1 & \cdots & \tilde{a}_{2 n} \\
\vdots & \vdots & \ddots & \vdots \\
1 / \tilde{a}_{1 n} & 1 / \tilde{a}_{2 n} & \cdots & 1
\end{array}\right]
$$

\section{Step 3: Aggregate the decision makers' preferences.}

The pairwise comparison matrices of decision makers' preferences are aggregated using equation below:

$$
\tilde{a}_{i j}=\left(\tilde{a}_{i j}^{1} \times \tilde{a}_{i j}^{2} \times \ldots \times \tilde{a}_{i j}^{n}\right)^{1 / k}
$$

where $k$ is the number of decision makers and $i=1,2, \ldots m ; j=1,2, \ldots n$.

Step 4: Defuzzify the fuzzy numbers of aggregation's result of decision makers' preferences.

The intuitive multiple centroid (IMC) defuzzification is extension from the classical vectorial centroid method for fuzzy numbers that proposed by [9], [10]. The concept is similar like the other centroid methods, to find the best centre point of fuzzy numbers that represent in crisp values or single values. Comparing to other centroid methods, IMC produces the appropriate way to get the output values that are more intelligent manner, easy to compute, more balance and consider all possible cases of fuzzy numbers. The IMC formula can be summarised as follows:

$$
I M C_{\tilde{\tilde{A}}}\left(\tilde{x}_{\tilde{A}}, \tilde{y}_{\tilde{A}}\right)=\left(\frac{2\left(a_{1}^{U}+a_{1}^{L}+a_{4}^{U}+a_{4}^{L}\right)+7\left(a_{2}^{U}+a_{2}^{L}+a_{3}^{U}+a_{3}^{L}\right)}{36}, \frac{7}{36}\left(h_{\tilde{A}}^{U}+h_{\tilde{A}}^{L}\right)\right)
$$

Step 5: Compute the centroid index of intuitive multiple centroid of $\left(\tilde{x}_{\tilde{A}}, \tilde{y}_{\tilde{A}}\right)$ with vertices $\alpha_{\alpha, \underline{\alpha}}, \beta_{\bar{\beta}, \underline{\beta}}$ and $\gamma_{\bar{\gamma}, \underline{\underline{\alpha}}}$.

Centroid index of intuitive multiple centroid can be generated using Euclidean Distance by [11] as:

$$
R(\tilde{A})=\sqrt{\tilde{x}^{2}+\tilde{y}^{2}}
$$


Step 6: Compute the criteria values as weightage for alternatives' evaluation using consistent fuzzy preference relations.

Consistent fuzzy preference relations was proposed by [12] for constructing the decision matrices of pairwise comparisons based on additive transitivity property. Referring to [13], a fuzzy preference relation $R$ on the set of the criteria or alternatives $A$ is a fuzzy set stated on the Cartesian product set $A \times A$ with the membership function $\mu_{R}: A \times A \rightarrow[0,1]$. The preference relation is denoted by $n \times n$ matrix $R=\left(r_{i j}\right)$ where $r_{i j}=\mu_{y}\left(a_{i}, a_{j}\right) \quad \forall i, j \in\{1, \ldots, n\}$. The preference ratio, $r_{i j}$ of the alternative $a_{i}$ to $a_{j}$ is determined by

$$
r_{i j}= \begin{cases}0.5 & a_{i} \text { is different to } a_{j} \\ (0.5,1) & a_{i} \text { is preferred than } a_{j} \\ 1 & a_{i} \text { is absolutely preferred than } a_{j}\end{cases}
$$

The preference matrix $R$ is presumed to be additive reciprocal, $p_{i j}+p_{j i}=1, \forall i, j \in\{1, \ldots, n\}$. Several propositions are associated to the consistent additive preference relations as follows:

Proposition 1 [14]: Consider a set of criteria or alternatives, $X=\left\{x_{1}, \ldots, x_{n}\right\}$, and associated with a reciprocal multiplicative preference relation $A=\left(a_{i j}\right)$ for $a_{i j} \in\left[\frac{1}{9}, 9\right]$. Then, the corresponding reciprocal fuzzy preference relation, $P=\left(p_{i j}\right)$ with $p_{i j} \in[0,1]$ associated with $A$ is given by the equation

$$
p_{i j}=g\left(a_{i j}\right)=\frac{1}{2}\left(1+\log _{9} a_{i j}\right)
$$

Generally, if $a_{i j} \in\left[\frac{1}{n}, n\right]$, then $\log _{n} a_{i j}$ is used, in particular, when $a_{i j} \in\left[\frac{1}{9}, 9\right]$; $\log _{9} a_{i j}$ is considered as in the above proposition because $a_{i j}$ is between $\frac{1}{9}$ and 9. If $a_{i j}$ is between $\frac{1}{7}$ and 7 , then $\log _{7} a_{i j}$ is used.

Proposition 2 [14]: For a reciprocal fuzzy preference relation $P=\left(p_{i j}\right)$, the following statements are equivalent.

$$
\begin{aligned}
& \text { (i) } p_{i j}+p_{j k}+p_{k i}=\frac{3}{2}, \forall i, j, k \\
& \text { (ii) } p_{i j}+p_{j k}+p_{k i}=\frac{3}{2}, \forall i<j<k
\end{aligned}
$$


Proposition 3 [14]: For a reciprocal fuzzy preference relation $P=\left(p_{i j}\right)$, the following statements are equivalent

$$
\begin{aligned}
& \text { (i) } p_{i j}+p_{j k}+p_{k i}=\frac{3}{2}, \forall i<j<k \\
& \text { (ii) } p_{i(i+1)}+p_{(i+1)(i+2)}+\ldots+p_{(j-1) j}+p_{j i}=\frac{j-i+1}{2}, \forall i<j
\end{aligned}
$$

Proposition 3 is crucial because it can be used to construct a consistent fuzzy preference relations form the set of $n-1$ values $\left\{p_{12}, p_{23}, \ldots, p_{n-1}\right\}$. A decision matrix with entries that are not in the interval [0,1], but in an interval $[-c, 1+c], c>0$, can be obtained by transforming the obtained values using a transformation function that preserves reciprocity and additive consistency with the function

$$
f:[-c, 1+c] \rightarrow[0,1], f(x)=\frac{(x+c)}{(1+2 c)}
$$

\section{Step 7: Ranking evaluation of alternatives using fuzzy TOPSIS}

Concept of TOPSIS method originally proposed by [15]. They claim that the alternative should not be chosen based on having the shortest distance from the positive ideal reference point (PIRT) only, but also have the longest distance from the negative ideal reference point (NIRP) in solving the MCDM problems. Here, the extension of fuzzy TOPSIS is illustrated differs from others in terms of the usage of defuzzification method, normalization process and ranking. The fuzzy decision matrix is constructed and the linguistic terms from fuzzy numbers are used to evaluate the alternatives with respect to criteria. Then, aggregate DMs' preferences:

$$
\begin{aligned}
& \overline{D M}=\begin{array}{c}
A_{1} C_{1} \\
A_{2} \\
\vdots \\
A_{m}
\end{array}\left[\begin{array}{cccc}
\tilde{x}_{11} & \tilde{x}_{22} & \cdots & C_{n} \\
\tilde{x}_{21} & \tilde{x}_{22} & \cdots & \tilde{x}_{1 n} \\
\vdots & \vdots & \ddots & \vdots \\
\tilde{x}_{m 1} & \tilde{x}_{m 1} & \cdots & \tilde{x}_{m n}
\end{array}\right] \\
& i=1,2, \ldots, m ; \quad j=1,2, \ldots, n \quad, \tilde{x}_{i j}=\frac{1}{K}\left(\tilde{x}_{i j}^{1} \oplus,,, \oplus \tilde{x}_{i j}^{k} \oplus,,, \tilde{x}_{i j}^{K}\right)
\end{aligned}
$$

where $x_{i j}$ is the performance rating of alternatives, $A_{i}$ with respect to criterion $C_{i}$ evaluated by $k$ th experts and $\tilde{x}_{i j}=\left(a_{1}^{k}, a_{2}^{k}, a_{3}^{k}, a_{4}^{k} ; h^{k}\right)$. Fuzzy decision matrix is weighted and normalised. Then, defuzzify the standardised generalised fuzzy numbers into coordinate form, $(\tilde{x}, \tilde{y})$. The weighted fuzzy normalised decision matrix is denoted by $\tilde{V}$ as depicted below:

$$
\tilde{V}=\left[\tilde{v}_{i j}\right]_{m \otimes n} ; \quad i=1,2, \ldots, m ; \quad j=1,2, \ldots, n
$$

where

$$
\tilde{v}_{i j}=\tilde{x}_{i j} \times \tilde{w}_{j}
$$


Normalised each generalised trapezoidal fuzzy numbers into standardised generalised fuzzy numbers. The weights from consistent fuzzy preference relations are adopted here. Defuzzify the standardised generalised fuzzy numbers using intuitive multiple centroid, then translate them into the index point. Use the new point of $y_{\tilde{A}_{i}^{*}}$ to compute the index centroid point of standardised generalised trapezoidal fuzzy numbers using Euclidean distance equation: $R\left(\tilde{A}_{i}^{*}\right)=\sqrt{\tilde{x}_{i}^{*^{2}}+\tilde{y}_{i}^{S^{2}}}$. Determine the fuzzy positive-ideal solution (FPIS) and fuzzy negative-ideal solution (FNIS). Referring to normalise trapezoidal fuzzy weights, the FPIS, $A^{+}$represents the compromise solution while FNIS, $A^{-}$represents the worst possible solution. The range belong to the closed interval [0,1]. The FPIS $A^{+}$(aspiration levels) and FNIS $A^{-}$(worst levels) as following below:

$$
A^{+}=[1,1,1,1 ; 1][1,1,1,1 ; 0.9] \quad A^{-}=[-1,-1,-1,-1 ; 1][-1,-1,-1,-1 ; 0.9]
$$

The FPIS, $A^{+}$and FNIS, $A^{-}$can be obtained by centroid method for $\left(x_{A^{+}}, y_{A^{+}}\right)$and $\left(x_{A^{-}}, y_{A^{-}}\right)$.

The distance $\tilde{d}_{i}^{+}$and $\tilde{d}_{i}^{-}$of each alternative from formulation $A^{+}$and $A^{-}$can be calculated by the area of compensation method:

$$
\begin{aligned}
& \bar{d}_{i}^{+}\left(\tilde{v}_{i j}, \tilde{v}_{j}^{+}\right)=\sqrt{\left(x_{\tilde{A}_{i}^{*}}-x_{A^{+}}\right)^{2}+\left(y_{\tilde{A}_{i}^{*}}-y_{A^{+}}\right)^{2}} \\
& \bar{d}_{i}^{-}\left(\tilde{v}_{i j}, \tilde{v}_{j}^{-}\right)=\sqrt{\left(x_{\tilde{A}_{i}^{*}}-x_{A^{-}}\right)^{2}+\left(y_{\tilde{A}_{i}^{*}}-y_{A^{-}}\right)^{2}}
\end{aligned}
$$

Find the closeness coefficient, $C C_{i}$ and improve alternatives for achieving aspiration levels in each criteria. Notice that the highest $C C_{i}$ value is used to determine the rank.

$$
\overline{C C}_{i}=\frac{\bar{d}_{i}^{-}}{\bar{d}_{i}^{+}+\bar{d}_{i}^{-}}=1-\frac{\bar{d}_{i}^{+}}{\bar{d}_{i}^{+}+\bar{d}_{i}^{-}}
$$

where, $\frac{\bar{d}_{i}^{-}}{\bar{d}_{i}^{+}+\bar{d}_{i}^{-}}$is satisfaction degree in ith alternative and $\frac{\bar{d}_{i}^{+}}{\bar{d}_{i}^{+}+\bar{d}_{i}^{-}}$is fuzzy gaps degree in ith alternative.

Fuzzy gap should be improvised for reaching aspiration levels and get the best mutually beneficial strategy from among a fuzzy set of feasible alternatives.

Step 8: Validation process using sensitivity analysis

The results of fuzzy MCDM models are importantly needed to validate using sensitivity analysis method to analyse the quality and how robustness of fuzzy MCDM model to reach a right decision under different conditions. In this paper, sensitivity analysis that proposed by [6] is utilised for validation purposes. 


\section{Case Study}

This section illustrates a numerical example for proposed hybrid fuzzy MCDM methodology based on real case study for staff recruitment problem in MESSRS SAPRUDIN, IDRIS \& CO firm in Malaysia. The legal company plan to hire the best candidate for executive post in several aspects which there are three decision makers (DMs) DM1, DM2, and DM3 of a firm and four alternatives or candidates $x 1, x 2, x 3$ and $x 4$. Several criteria are considered which are: C1) Emotional steadiness, C2) Oration, C3) Personality, C4) Past experience and, C5) Self-confidence. This study simplifies the concept of attributes under fuzzy events. The values of attributes correspond to interval type-2 fuzzy sets. A comparative study was conducted to validate the results of the proposed model with established hybrid model which is fuzzy AHP - fuzzy TOPSIS proposed by [16].

Table 1. Ranking results of criteria for comparing study.

\begin{tabular}{ccccccc}
\hline Fuzzy MCDM model & \multicolumn{6}{c}{ Criteria weight values } \\
& $\boldsymbol{E S}$ & $\boldsymbol{O}$ & $\boldsymbol{P}$ & $\boldsymbol{P E}$ & $\boldsymbol{S}-\boldsymbol{C}$ & \\
\hline Fuzzy AHP - TOPSIS [16] & 0.087 & 0.364 & 0.044 & 0.34 & 0.164 & $\mathrm{O}>\mathrm{PE}>\mathrm{S}-\mathrm{C}>\mathrm{ES}>\mathrm{P}$ \\
Proposed Model & 0.1172 & 0.2672 & 0.1190 & 0.2747 & 0.2219 & $\mathrm{PE}>\mathrm{O}>\mathrm{S}-\mathrm{C}>\mathrm{P}>\mathrm{ES}$ \\
\hline
\end{tabular}

Table 1 represents the criteria weight and ranking results between established fuzzy AHP - fuzzy TOPSIS [16] and proposed model. Based on decision makers' evaluation; Past experience, Oration and Self-confidence criteria play important aspects in recruiting new staff since the weight are greater than 0.2 respectively. These results of criteria's weights are implemented in following phase to evaluate for alternatives selection. The established fuzzy AHP - fuzzy TOPSIS model [16] produces different ranking results with rank Oration > Past Experience > Selfconfidence $>$ Emotion Steadiness $>$ Personality.

Table 2. Ranking results of alternatives for comparing study

\begin{tabular}{cccccc}
\hline \multirow{2}{*}{ Fuzzy MCDM model } & \multicolumn{6}{c}{ Alternatives ranking values, $\boldsymbol{C C} \boldsymbol{i}$} & \multirow{2}{*}{ Ranking results } \\
\cline { 2 - 5 } & Alt1 & Alt2 & Alt3 & Alt4 & \\
\hline Fuzzy AHP - TOPSIS [16] & 0.5497 & 0.5543 & 0.5616 & 0.5413 & $\mathrm{C} 3>\mathrm{C} 2>\mathrm{C} 1>\mathrm{C} 4$ \\
Proposed Model & 0.7422 & 0.7823 & 0.83 & 0.6964 & $\mathrm{C} 3>\mathrm{C} 2>\mathrm{C} 1>\mathrm{C} 4$ \\
\hline
\end{tabular}

Table 2 depicts the alternatives/ candidates ranking results for $C C_{i}$ values. The proposed model evaluates candidate 3 as the highest rank with 0.83 followed by candidate 2 , candidate 1 and candidate 4 for the last rank. The results reveal that the candidate 3 is most suitable for this recruitment post. The established model [16] produces same ranking results for alternatives with the proposed model. Even, this model gives same ranking to proposed model, but the gaps of $C C i$ values between each candidate are too small. Those ranking results by [16] will easily affected if the weightage of criteria are slightly changed. This can be evaluated by sensitivity analysis in studying how consistent and robust the model. In the context of sensitivity 
analysis evaluation, it presents that the proposed hybrid fuzzy MCDM model is definitely consistent even the weights of criteria are changed with several percentages. From the consistency results, the proposed hybrid fuzzy MCDM model is recommended to deal with bigger case study in real world phenomena in order to solve human based decision making problems under fuzzy environment.

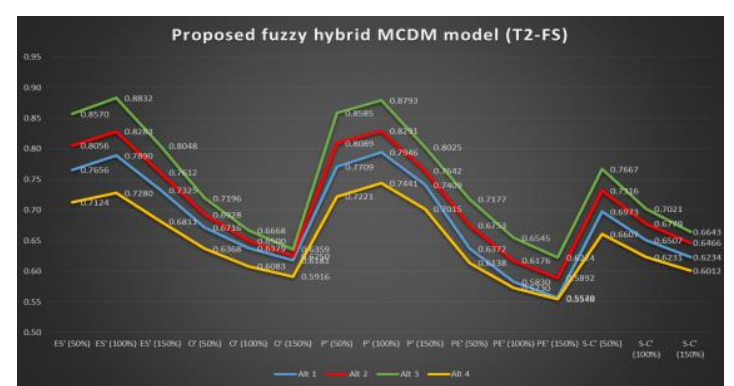

Fig. 2. Sensitivity analysis results by varying the weights of the criteria by proposed model.

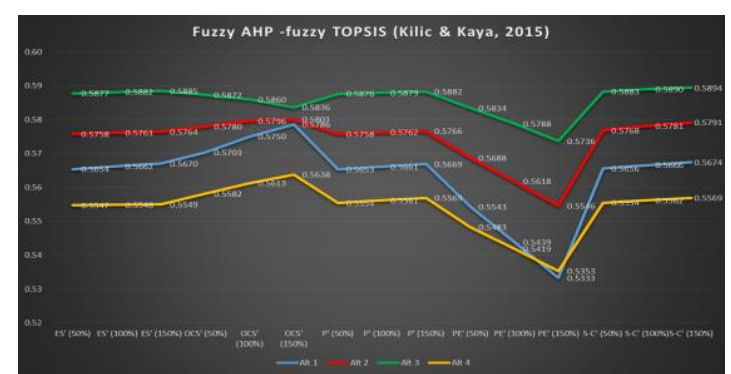

Fig. 3. Sensitivity analysis results by varying the weights of the criteria by [16].

Fig. 2 illustrates the analysis results of changing the criteria weights for proposed model. It presents that when the weights of the criteria change, the values of the $C C_{i}$ vary slightly. As can be seen from Fig. 3, the values and patterns of changes of $C C_{i}$ are too small compare to the proposed model. The ranking values between alternative to other alternative are too small. That's mean that the gap are small to represent the assessment status of acceptance. This is depicted that the proposed model is good in robustness than established model [16].

\section{Conclusion}

This study has brought out the idea and concept regarding the fuzzy MCDM model that consist of consistent preference relations and fuzzy TOPSIS using intuitive multiple centroid (IMC) defuzzification method based on interval type-2 fuzzy sets. The development of IMC provides efficient computational defuzzification procedures for fuzzy sets. It presents in simple formulae that based on the perspective of analytic geometric principles. In developing an intuitionistic defuzzification, a novel manner 
of computing intuitive multiple centroid method has capability in dealing with all possible cases of interval type-2 fuzzy numbers. The development of fuzzy MCDM model provides better selection in human based decision making problems where at the same capable to deal with uncertainty in human judgment. Due to access information and availability of the uncertain data, it is hard to make right decision. In this sense, it is important to improvise the techniques or models form the classical one, adding intuitive reasoning and human subjectivity. As consequence, the proposed model is developed to design the robust and consistent methodology in order to give the most promising alternatives with respect to the resources. Therefore, this proposed model can be further proceeded in order to make some contributions by considering complicated case studies drawn for diverse fields crossing human based decision making problems.

\section{References}

[1] G. J. Klir, U. St. Clair, and B. Yuan, Fuzzy set theory: foundations and applications, Internatio. Prentice Hall, 1997.

[2] C. Wagner and H. Hagras, "Uncertainty and type-2 fuzzy sets and systems," Comput. Intell. (UKCI), 2010 UK Work., pp. 1-5, 2010.

[3] N. N. Karnik and J. M. Mendel, "Centroid of a type-2 fuzzy set," Informayion Sci., vol. 132, pp. 195-220, 2001.

[4] N. Mogharreban and L. F. Dilalla, "Comparison of defuzzification techniques for analysis of noninterval data," Annu. Conf. North Am. Fuzzy Inf. Process. Soc. - NAFIPS, no. 1, pp. 257-260, 2006.

[5] N. N. Karnik and J. M. Mendel, "Centroid of a type-2 fuzzy set," Inf. Sci. (Ny)., vol. 132, no. 1-4, pp. 195-220, Feb. 2001.

[6] A. Amini and A. Alinezhad, "Sensitivity Analysis of TOPSIS Technique: The Results of Change in the Weight of One Attribute on the Final Ranking of Alternatives," J. Optim. Ind. Eng., vol. 7 , no. 2011, pp. 23-28, 2011.

[7] H. Deng, "Comparing and ranking fuzzy numbers using ideal solutions," Appl. Math. Model., vol. 38, no. 5-6, pp. 1638-1646, Mar. 2014.

[8] Y. Gong, N. Hu, J. Zhang, G. Liu, and J. Deng, "Multi-attribute group decision making method based on geometric Bonferroni mean operator of trapezoidal interval type-2 fuzzy numbers," Comput. Ind. Eng., vol. 81, pp. 167-176, 2015.

[9] K. M. N. Ku Khalif and A. Gegov, "Generalised Fuzzy Bayesian Network with Adaptive Vectorial Centroid," in 16th world congress of the international fuzzy systems association (IFSA) and the 9th conference of the European society for fuzzy logic and technology (EUSFLAT), 2015, pp. 757-764.

[10] K. M. N. Ku Khalif and A. Gegov, "Bayesian Logistic Regression using Vectorial Centroid for Interval Type-2 Fuzzy Sets," in 7th International Joint Conference on Computational Intelligence (IJCCI 2015), 2015, pp. 69-79.

[11] C.-H. Cheng, "A new approach for ranking fuzzy numbers by distance method,” Fuzzy Sets Syst., vol. 95 , no. 3, pp. 307-317, May 1998.

[12] E. Herrera-Viedma, F. Herrera, F. Chiclana, and M. Luque, "Some issues on consistency of fuzzy preference relations,” Eur. J. Oper. Res., vol. 154, no. 1, pp. 98-109, 2004.

[13] N. H. Kamis, K. Abdullah, H. Mohamed, S. Sudin, and W. Z. A. W. Ishak, "Decision making models based on consistent fuzzy preference relations with different defuzzification methods," 2011 IEEE Colloq. Humanit. Sci. Eng. CHUSER 2011, no. Chuser, pp. 845-850, 2011.

[14] T. C. Wang and Y. H. Chen, "Applying consistent fuzzy preference relations to partnership selection," Omega, vol. 35, no. 4, pp. 384-388, 2007.

[15] C.-L. Hwang and K. Yoon, Multiple Attribute Decision Making. New York: Springer, 1981.

[16] M. Kiliç and İ. Kaya, "Investment project evaluation by a decision making methodology based on type-2 fuzzy sets," Appl. Soft Comput., vol. 27, no. 2015, pp. 399-410, 2015. 